\title{
A perceção dos profissionais de saúde sobre fontes de informação e sua influência na relação terapêutica
}

\section{The perception of health professionals about information sources and their influence on the therapeutic relationship}

\author{
Cristina Vaz de Almeida*, Sónia Pedro Sebastião**
}

*ISCSP, Universidade de Lisboa; CAPP/FCT

** ISCSP, Universidade de Lisboa; CAPP/FCT

Resumo

\begin{abstract}
Este estudo tem como objetivo a aferição da perceção dos profissionais de saúde sobre a importância das fontes de informação para a relação terapêutica. Recorreu-se a uma metodologia qualitativa, através da realização de focus group, baseado num questionário semiestruturado, aplicado a nove especialistas em literacia em saúde e profissionais das áreas da saúde em Portugal. Os participantes enfatizaram a responsabilidade do profissional de saúde como fonte especialista e credível para o fornecimento de informação e direcionamento do paciente quando o mesmo recorre a fontes menos credíveis como a internet. Foi ainda destacado o papel dos pares (outros profissionais de saúde) e dos pacientes que prestam depoimento sobre a sua experiência (por exemplo, doentes crónicos).
\end{abstract}

Palavras-chave: comunicação em saúde, credibilidade das fontes, teoria situacional da resolução de problemas, literacia em saúde

Abstract

This study aimed to assess the perception of health professionals about the importance of information sources for the therapeutic relationship. A qualitative methodology was used, through the moderation of a focus group, based on a semi-structured questionnaire, applied to nine Portuguese health professionals interested in health literacy. Participants emphasized that health professionals have an increased responsibility in the therapeutic relationship, particularly in the management of communicational aspects, as well as, credible source of information that besides providing reliable information, must also direct patients when they use sources of information less reliable like the internet. It also highlighted the role of peers (other health professionals) and patients reporting on their experience (e.g. chronical disease patients).

Key words: health communication, credibility of sources, situational theory of problem resolution, health literacy

\section{Introdução}

Numa sociedade em rede e da informação torna-se necessário aceder, selecionar, dar sentido, compreender e usar a informação de modo a que a mesma seja adjuvante na resolução de um problema. Assim sendo, não basta existir informação, nem que a mesma seja comunicada, é também necessário que esta seja apreendida e utilizada na melhoria e manutenção de uma situação favorável ao recetor.

A Organização Mundial da Saúde (1986) vê a comunicação como uma estratégia-chave no processo de disseminação de informação sobre temas centrais à saúde das populações. Salvaguardando que as 
estratégias e programas de promoção da saúde deverão ser adaptados às necessidades locais e às possibilidades de cada país e região, considerando os diferentes sistemas sociais, culturais e económicos. Atendendo à importância da comunicação em saúde, mas conscientes da vastidão do campo, neste artigo, analisa-se a relação terapêutica entre profissionais da saúde e pacientes como um elemento fundamental do processo comunicativo em saúde. Assim sendo, e seguindo a tipologia de Berlo (1985), neste processo existe: emissor/codificador/fonte (profissional da área da saúde), a mensagem (informação sobre doença e terapêutica, comportamento para a saúde), o canal (ar, telefone, meios eletrónicos), recetor/descodificador (paciente, familiar, cuidador). Consideram-se os profissionais da área da saúde como as principais fontes/codificadores da informação que permitem a prossecução da saúde e da educação para a saúde. Por outras palavras, são o elemento emissor de informação numa relação terapêutica. Esta é uma relação interpessoal comunicacional e colaborativa entre profissional e paciente (ou cuidador), num determinado espaço e tempo, que visa a valorização e a capacitação da pessoa, para que esta compreenda e possa aderir, de forma autónoma, às instruções em saúde (mensagem), com vista aos melhores resultados em saúde (Vaz de Almeida, 2016).

No campo sensível da saúde, a importância de fontes de informação de confiança, credíveis (Cassel, 1985, p. 157), e sobretudo competentes, é fundamental face às consequências nefastas que má informação pode ter na vida do indivíduo e até em termos de saúde pública. Se numa relação terapêutica, o emissor é o profissional de saúde, na adesão à terapêutica prescrita outros fatores precisam de atenção devido à presença de fontes de informação indiretas à relação terapêutica, mas que podem intervir e causar "ruído" na mesma. Entre estes fatores identificamos junto do público recetor (pacientes) problemas de literacia em saúde e o recurso a fontes de informação que não os profissionais da área da saúde.

Com o objetivo de aferir a perceção dos profissionais de saúde sobre a importância das fontes de informação para a relação terapêutica, considera-se em termos teóricos: o conceito de literacia em saúde, evidenciando a importância da comunicação e das fontes de informação para o seu aumento; a teoria situacional dos públicos (Grunig \& Hunt, 1984) e a definição dos públicos inativos (Hallahan, 2000) para caracterizar os públicos envolvidos nesta relação em termos de conhecimento e envolvimento; a teoria situacional de resolução dos problemas (Kim \& Grunig, 2011), assim como, a teoria cognitiva social de Bandura (1986) mostrando a importância da ação comunicativa e da autoeficácia para a relação terapêutica e subsequente resolução de um problema.

\section{Comunicação e Literacia em Saúde}

De acordo com os princípios de Ottawa (Organização Mundial da Saúde, 1986), as condições básicas e as expetativas face à saúde só podem ser asseguradas através de ação coordenada de todos os intervenientes, entre eles: governos e autarquias; sectores da saúde, social e económico; organizações não-governamentais e de voluntários; empresas e comunicação social; assim como, as comunidades, as famílias e os indivíduos. Estes intervenientes devem procurar educar-se mutuamente sobre questões de saúde e desenvolver formas de comunicar a informação, até porque e segundo a Organização Mundial da Saúde: a comunicação em saúde (health communication) é uma estratégia chave para informar o público sobre preocupações com a saúde e para manter assuntos de saúde na agenda pública. O uso dos media e de outras inovações 
tecnológicas para disseminar informação de saúde útil ao público, aumenta a consciência sobre aspetos específicos de saúde individual e coletiva, assim como, sobre a importância dos desenvolvimentos em saúde (World Health Organization, 1998, p. 8). Todavia, estes mesmos meios, além de não serem inclusivos pois não existe igualdade de acesso, podem veicular informação incorreta e induzir os indivíduos em erro, tornando-se oponentes no processo comunicativo entre o profissional da área da saúde e o paciente.

A comunicação em saúde tanto inclui o estudo e aplicação da criação e disseminação de informações relacionadas com a saúde; como das interações relacionadas com saúde entre atores e instituições sociais e individuais e os seus efeitos em diferentes públicos, abrangendo indivíduos, grupos comunitários e instituições. Por isso, a investigação sobre este tema pode focar três níveis: 1) o individual, que inclui o estudo dos efeitos da comunicação sobre as cognições e sobre os comportamentos de saúde; assim como, o estudo da influência nos resultados de saúde das interações interpessoais entre pacientes, familiares e provedores, e respetivas redes sociais; 2 ) o organizacional, em que são consideradas a comunicação pública dos sistemas de saúde e dos media e respetivas influências na saúde dos indivíduos e da população; e 3) o societal cujo foco está nas mudanças sociais de grande escala e no papel da comunicação nessas mudanças (Viswanath, 2008, p. 2075).

Todos os intervenientes podem ser públicos na comunicação em saúde, assumindo, conforme as situações, o papel de emissores e recetores de mensagens relevantes para esta área. A comunicação usada pelo profissional da área da saúde, outras questões biopsicossociais relacionadas com o perfil dos recetores (tais como: baixos estratos socioeconómicos, minorias étnicas, idosos, incapacidades cognitivas, doenças crónicas, baixos níveis de escolaridade e habilidades) e a baixa literacia em saúde podem afetar, de forma adversa, a compreensão do paciente sobre o seu estado de saúde e a sua adesão terapêutica (Cegala, McNeilis \& McGee, 1995; Kripalani, et al., 2008; Chinn \& McCarthy, 2012).

Baixos níveis de literacia em saúde estão normalmente associados a baixos níveis educacionais, de acesso a informação e provocam a diminuição da autonomia do indivíduo (Espanha, Ávila, \& Mendes, 2016). De acordo com Cutilli (2010), os profissionais de saúde acreditam que o aumento da literacia em saúde contribui para melhores resultados da sua atividade, isto é, contribui para a melhoria das atitudes e comportamentos dos indivíduos em relação às recomendações em saúde.

A literacia em saúde pode ser um fator chave para ativar o ser humano para uma melhor compreensão sobre o universo que o rodeia, em particular o contexto complexo de saúde. Através de um processo de comunicação, "feito à medida", promove-se a capacitação com vista a uma tomada de decisões corretas por parte do indivíduo em relação à sua saúde, à saúde dos seus dependentes e, até, à saúde pública.

Neste trabalho, a literacia em saúde é entendida como um constructo, complexo, que pela sua dinâmica, continua em evolução e relaciona-se com o desenvolvimento das competências dos indivíduos para melhor acederem, usarem, gerirem e compreenderem o sistema e as informações sobre saúde, para poderem tomar decisões e manter (ou melhorar) a sua saúde e a dos seus dependentes (Sørensen, et al., 2012). Os dados do Questionário Europeu de Literacia em Saúde (Sørensen, et al., 2012), composto por 47 questões integrando os domínios dos cuidados de saúde, promoção da saúde e prevenção da doença, com quatro níveis de processamento da informação nas vertentes do acesso, compreensão, avaliação e utilização da informação em saúde, demonstram que as pessoas na Europa têm níveis baixos de literacia em saúde. Também em Portugal, $61 \%$ da população inquirida apresentava um nível de literacia geral em saúde problemático ou inadequado, situando-se a média dos nove países avaliados em 49,2\% (Sørensen, et al., 
2012; Saboga-Nunes, 2014). A baixa literacia em saúde da população portuguesa acarreta um problema de falta evidente na compreensão, assim como, de acesso, participação e avaliação da informação e dos sistemas de saúde, com custos individuais e sociais (Saboga-Nunes, 2014; Espanha, Ávila, \& Mendes, 2016). Perante este problema, atenta-se no primeiro nível da literacia em saúde, isto é, no acesso a informação, procurando perceber que fontes de informação podem ser adjuvantes ou oponentes na relação e adesão terapêuticas. As fontes de informação são consideradas como redes de indivíduos, grupos ou instituições que fornecem dados e recursos sobre diversos assuntos que podem influenciar as atitudes e comportamentos dos indivíduos. Estas fontes são estruturas de comunicação, canais de mediação informativa que englobam redes interpessoais, organizacionais e mediáticas, com um papel no processo de formação de opinião.

\section{Fontes de Informação}

A alteração do espectro mediático provocada pela evolução tecnológica e pelo surgimento da internet e de numerosas plataformas digitais multiplicou exponencialmente o número de fontes de informação e as possibilidades de eco de informações não controladas pelas estruturas dominantes da sociedade.

Uma fonte de informação é alguém (pessoa) ou algo (meio) que providencia dados ou conhecimento sobre um tema específico para satisfação da necessidade de informação de outrem. Segundo Pinto (2000), as fontes podem ser pessoas, grupos, instituições sociais ou vestígios - falas, documentos, dados - por aqueles preparados, construídos, deixados; remetendo para posições e relações sociais, para interesses e pontos de vista, para quadros espácio-temporalmente situados (Pinto, 2000, p. 278).

A fonte de informação pode ter três bases relacionadas com os laços existentes entre emissor e recetor: pessoal (família e colegas originalmente estudados por Katz e Lazarsfeld, 1955) decorrente de relações primárias, marcada por laços fortes e estreitos, e caracterizada pela confiança, credibilidade e proximidade; e uma impessoal (Mutz, 1998) que emana de relações formais e mediadas, principalmente estabelecidas entre o indivíduo e os media, ou outras organizações que respondam à necessidade ampla de informações. Fontes impessoais de informação são caracterizadas por laços fracos e são dominadas por especialistas. Apesar dos especialistas poderem ser fontes mais credíveis, poderão ser preteridas face à "distância social" da pessoa que procura a informação. Com a sociedade da informação é possível identificar uma terceira fonte de informação - a web - que pode ser definida como híbrida, uma vez que reúne laços fracos e fortes e pode surgir a partir de fontes primárias e organizacionais.

De acordo com os atributos de fidelidade de Berlo (1985), existe uma hierarquia de fontes. A primazia das fontes associa-se aos incentivos, ao poder, à credibilidade e à proximidade geográfica e social. Considerase que é a informação credível sobre diversos assuntos que permite ao indivíduo tomar decisões sustentadas e racionais. A credibilidade da mensagem pode ser definida como um conjunto de atributos que torna 0 conteúdo - ou a sua fonte - valorizado por quem é exposto à mensagem. Para Cassell (1985, p. 157), a credibilidade é o grau em que o emissor é percebido pelo recetor como fonte fidedigna e competente de informação. Neste sentido, a credibilidade envolve dois atributos fundamentais: a expertise da fonte, i.e., o seu conhecimento e precisão na abordagem ao tema; e a confiabilidade relacionada com as intenções da fonte (Rouner, 2008, p. 1041). 
Na área da saúde, a informação tem de ser credível para garantir o compromisso e a adesão do paciente e a credibilidade será maximizada se o profissional for considerado um especialista pelo paciente (Jackson, 1992, p 201). Por exemplo, na escolha dos planos de saúde, os pacientes colocam muita confiança no seu médico, família e amigos (Isaacs,1996, p. 38).

Enquanto fonte, o profissional usa uma linguagem que deve ser compreendida pelo paciente (linguagem simples). Mas o profissional de saúde não é a única fonte de informação consultada pelos pacientes sobre saúde. Perante a panóplia de fontes existentes, nem sempre a informação é correta. As razões para a pesquisa de informação em saúde são multifatoriais, sendo a principal responder a uma preocupação de saúde (53\%), seguindo-se a tentativa de seguir um estilo de vida saudável (31\%) ou ajudar outras pessoas (19\%) (Mukherjee \& Bawden, 2012). Os fatores como a necessidade de se ter conhecimento, o ambiente e o contexto onde a informação é necessária influenciam também a forma como esta informação é recolhida, especialmente as fontes privilegiadas e a frequência da sua utilização (Mukherjee \& Bawden, 2012).

As razões para a pesquisa de informação em saúde pelo público em geral são variadas e esta ocorre sobretudo em resposta a uma necessidade ou falta de conhecimento (Mukherjee \& Bawden, 2012), devendo olhar-se para as fontes de informação como parte da estratégia para abordar a literacia em saúde (Norman \& Skinner, 2006).

Em suma, as fontes para procurar informação em saúde são várias (ver figura 1) e podemos elencar a Internet, livros, amigos e familiares, centros de saúde e instituições afins, linhas telefónicas, folhetos, notícias e testemunhos de pacientes.

Figura 1: Fontes de Informação na área da saúde

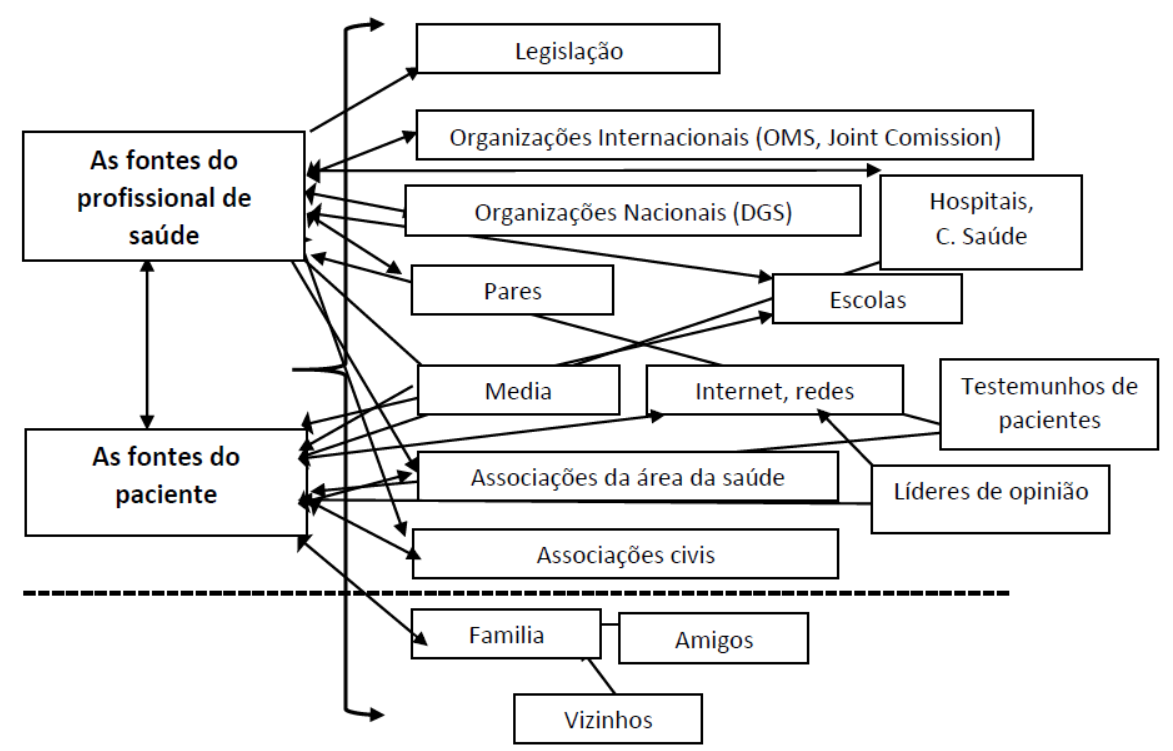

Fonte: Elaboração Própria

As fontes eletrónicas como a internet e as redes sociais online são parte integrante dos contextos de informação em saúde, sendo bastante utilizados pela população em geral, e em particular pelos adolescentes (Andreassen, et al., 2007, Ghaddar, et al., 2012; Gray, et al., 2005; Norman \& Skinner, 2006; Stellefson, et al., 2011). Contudo mesmo que alguns estudos apontem a internet como uma das fontes de informação 
consideradas credíveis pelos pacientes e seja preferida devido à velocidade de acesso, disponibilidade e variedade da informação, tem de haver sempre um "acesso adequado" (Simou, 2016, p. 42). Só este tipo de acesso pode contribuir para aumentar a literacia em saúde e promover competências para a compreensão da informação e diminuição dos riscos associados a informação imprecisa.

Por outro lado, também os testemunhos dos pares, isto é, de outros pacientes que sofreram ou sofrem da mesma doença são valorizados. A doença faz emergir emoções, e, segundo Heisler (2006) estas trocas de experiência pelos pares funcionam porque não são hierárquicas, existe uma relação recíproca, uma partilha de experiências e conhecimentos com outros que enfrentaram ou enfrentam desafios semelhantes.

As emoções otimizam as decisões sociais e são como sistemas de comunicação que ajudam as pessoas a navegar e coordenar as interações sociais permitindo a criação e a manutenção de relações sociais saudáveis e produtivas (Keltner, Oatley, \& Jenkins, 2014). Neste sentido compreende-se que, apesar da subjetividade e parcialidade da visão de quem está a passar ou passou por uma doença, o certo é que as suas particularidades (Heisler, 2006, p. 35), a tornam credível aos ouvidos e à perceção de outros, sejam doentes, sejam os cuidadores formais em saúde.

Quando e como os indivíduos usam informações suplementares tem variações, e está associado a fatores como a literacia em saúde, o estado de saúde, a ocupação, o nível educacional, cognitivo, emocional, entre outros fatores que se refletem na confiabilidade maior ou menor das fontes com que contactam.

Em síntese, a revisão de literatura evidencia que a fonte de informação mais comum e confiável são os profissionais de saúde. As pessoas tendem a recorrer a outras fontes de informação de saúde (por exemplo: internet, família, amigos e outros media) para complementar as informações fornecidas por estes profissionais (e.g. Cutilli, 2010).

\section{Públicos, ação comunicativa e resolução de problemas}

A teoria situacional dos públicos - utilizada para identificar públicos estratégicos - procura explicar quando e porque comunicam os públicos, e de que forma a comunicação organizacional poderá ser mais ou menos eficaz, ou seja, a teoria situacional dos públicos procura identificar os comportamentos comunicativos dos públicos, normalmente, do ponto de vista da organização. Esta teoria combina e melhora as conceções de público de Dewey (1927) e de opinião pública de Blumer (1966), segundo as quais os públicos são componentes cruciais do processo democrático, pois reconhecem os problemas, organizam-se e atuam de modo a resolvê-los. Em última instância, as organizações - independentemente da sua área de atuação existem para e por causa dos públicos.

A teoria situacional dos públicos é referência em trabalhos dos investigadores da Teoria da Excelência, mas também em trabalhos de autores como Aldoory e Sha (2007); Chaiken (1980); Chew (2001); Kim, Ni e Sha (2008). Sendo também atualizada pela perspetiva de Hallahan (2000) que classifica os públicos de acordo com o seu envolvimento e conhecimento. Os públicos inativos distinguem-se dos públicos ativos pelo seu baixo nível de conhecimento sobre a organização e baixo envolvimento nas suas ações. Em relação aos públicos intermédios, Hallahan (2000) distingue os públicos conscientes com elevado nível de conhecimento sobre a organização e baixo envolvimento nas suas ações, uma vez que não são diretamente afetados pelas mesmas; e os públicos estimulados, com elevado envolvimento, mas pouco conhecimento. A estimulação 
destes públicos pode ser motivada pela experiência pessoal, pela informação veiculada pelos órgãos de comunicação social, pela opinião de um amigo ou pela atividade de grupos de pressão, partidos políticos e grupos ativistas (Hallahan, 2000). Embora a teoria situacional dos públicos permita a compreensão dos públicos em vários contextos, há autores que consideram que esta é limitada porque não explica o processo de aquisição de informação pelos públicos sobre um determinado assunto de uma organização, ou seja, não esclarece como os públicos se envolvem em conversas nas esferas públicas, nem quais as suas fontes de informação (e.g. Moon, Rhee, \& Yang, 2016, p. 5; Kim \& Grunig, 2011). No entanto, esta teoria evidencia que o consumo de informação se torna sistemático, quando os públicos avaliam a informação como resolvente dos seus problemas, isto é, comunicam mais quando percebem que o seu esforço é necessário e relevante (Grunig, 1997).

Decorrente da teoria situacional dos públicos (Grunig \& Hunt, 1984; Grunig \& Repper, 1992; Hunt \& Grunig, 1994) e acolhendo o criticismo de Hallahan (2000), Kim e Grunig (2011) propõem a teoria situacional da resolução de problemas. Esta teoria acrescenta a variável dependente da ação comunicativa na resolução de problemas e a variável independente do critério referente. Este é cognitivo e determinado por uma condição antecedente, de base social e associada a comportamentos passados da organização ou dos indivíduos que integram determinado público. Adicionalmente, os autores prevêem o conceito mediador - a motivação situacional na resolução de problemas - para relacionar fatores cognitivos, percetivos e traços pessoais que influenciam a extensão da necessidade e o uso da informação (Kim \& Grunig, 2011).

Os públicos investem na procura de informação e na ação comunicativa para resolver problemas, portanto, agem quando o esforço é percebido como necessário e relevante. 0 reconhecimento dos constrangimentos desencoraja o indivíduo que percebe a existência de obstáculos que limitam a sua capacidade de atuação sobre a situação (Grunig, 1997), ou, na terminologia de Bandura (1986), a autoeficácia. No campo da saúde, o aumento da autoeficácia leva o paciente a avaliar melhor a perceção do risco, a ter maior confiança na sua capacidade para evitar ameaças e a aumentar a intenção de adotar comportamentos saudáveis (Anderson \& McMillion, 1995). Se não existir um mecanismo que estimule a motivação situacional para a resolução do problema e afaste o constrangimento, manter-se-á a atitude passiva do indivíduo na aquisição, seleção e transmissão de informação (Kim \& Grunig, 2011).

Esta ação comunicativa tem, contudo, mais expressões que as consideradas na teoria situacional dos públicos. Ou seja, para além da aquisição (procura e processamento), poderá ocorrer transmissão (reencaminhamento e partilha) e seleção de informação (rejeição e aceitação) relativa a dado problema. Em suma, a teoria situacional da resolução de problemas: 1) analisa o porquê e como os públicos se tornam ativos em relação a determinado problema; e 2) como se envolvem em comportamentos comunicativos (Kim, et al., 2012).

Aplicando estes conceitos ao tema deste artigo e fazendo a analogia com os pacientes com baixa literacia em saúde, verifica-se que, o paciente com baixa literacia em saúde pode ser considerado como um público latente (Grunig \& Repper, 1992) e como um público inativo (Hallahan, 2000) com um nível baixo de conhecimento e de envolvimento. Prevendo-se na teoria da resolução de problemas um aumento da atividade comunicativa da pessoa que pretende resolver o problema, tal só ocorrerá quando a informação for percebida como útil. A atividade é assim determinada pela sua vontade e intenção, que acaba por espoletar uma ação proactiva (pesquisar, filtrar ou passar informação) ou reativa (receção, permissão ou partilha de informação). 
Embora as pessoas com baixa literacia em saúde, pelas suas características de enquadramento nos "públicos inativos" tenham baixo nível de conhecimento e de envolvimento, são importantes para o sistema e a sua inatividade não pressupõe falta de capacidade (Hallahan, 2000). A ativação destes públicos vai além destes conhecerem o problema, requerendo ainda que se combine mais conhecimento e envolvimento. Necessariamente são um grupo de pessoas com quem a organização de saúde, através dos seus profissionais, deseja manter uma relação.

Os profissionais da área da saúde, por sua vez, podem ser classificados como um público ativo. A intervenção do profissional da área da saúde poderá ser crítica, pois na relação terapêutica, este profissional é parte de um público com maior conhecimento do problema, maior envolvimento e mais baixo constrangimento para agir, podendo afirmar-se como o polo comunicativo (emissor) com controlo sobre a mensagem e com a informação necessária à resolução do problema. O profissional é um especialista que poderá orientar, influenciar decisões, persuadir o público latente ou inativo - o paciente com baixa literacia. Não obstante, a importância das competências comunicacionais dos profissionais da área da saúde (Williams, el al., 2002), a mensagem pode não ser compreendida pelos pacientes devido a falta de empatia entre os interlocutores; falta de interesse ou motivação; dificuldades cognitivas e de acesso à informação; não domínio do código usado na mensagem (por exemplo, jargão técnico) ou dificuldade de discernimento de informações contraditórias provenientes de várias fontes de informação, além das influências dos vários determinantes sociais da saúde (Wilkinson \& Marmot, 2003; Kickbusch, et al., 2013).

Perante este referente e na opinião do profissional da área da saúde, que outras fontes de informação podem então ser consideradas credíveis e adjuvantes da relação terapêutica contribuindo para a resolução do problema dos pacientes?

\section{Opções Metodológicas}

Este artigo tem como principal objetivo aferir a perceção dos profissionais de saúde sobre a importância das fontes de informação para a relação terapêutica. Recorreu-se, por isso, à realização de um focus group, "entrevista de grupo com vários participantes" (Bryman, 2012, p. 502) sobre um determinado tópico ou tema que é explorado em profundidade constituído por nove peritos em literacia em saúde e profissionais das áreas da saúde em Portugal. Todos estes profissionais de saúde têm uma preocupação ativa sobre a literacia em saúde e sobre os processos de capacitação dos profissionais de saúde para a melhoria da relação terapêutica. Atendendo ao tema em estudo, considerou-se que o focus group beneficiaria com a existência de uma base comum de interesse dos participantes na pesquisa do tópico, seguindo as recomendações de Ritchie e Lewis (2003, p.59). Neste âmbito, existe vantagem nesta seleção criteriosa do perfil destes profissionais de saúde pois, mesmo os que são docentes exercem clínica, desenvolvem com frequência interação com os pacientes e, por isso, detém contactos regulares com o ambiente real de consulta. Este e outros investimentos em estratégias de educação para a saúde, comunicação em saúde, com vista a uma maior abrangência das suas competências clínicas e competências relacionais, permitiram identificar um corpo de profissionais de saúde com níveis de percepção críticos e construtivos elevados em relação a esta temática da credibilidade das fontes. Ademais, por serem especialistas, não é necessário esclarecer conceitos 
ou dar explicações sobre o tema em estudo, situação que poderia condicionar o output da dinâmica de grupo.

Estes especialistas foram selecionados através da base de dados de profissionais da área da saúde que frequentaram a formação pós-graduada em Comunicação e Literacia em Saúde (das escolas ISPA e ESEL), e pela sua ativa intervenção nos domínios da literacia em saúde e da promoção da saúde. Depois de identificados, foram convidados por correio eletrónico e por telefone, tendo todos respondido afirmativamente e participado no focus group.

Em termos sociodemográficos, o grupo incluiu três especialistas do género masculino e seis especialistas do género feminino. Oito destes peritos desempenham funções profissionais ativas e uma especialista já se encontra aposentada, embora continue ativa na pesquisa e intervenção académica. As ocupações profissionais estão relacionadas com as várias áreas da saúde, nomeadamente: a oncologia, a medicina dentária, a enfermagem de reabilitação e a medicina tradicional chinesa. Cinco especialistas desempenham funções académicas, mas já tiveram larga experiência no terreno e contato direto com os pacientes. A tabela 1 apresenta a súmula dos participantes e respetiva codificação.

Tabela 1: Lista de participantes

\begin{tabular}{|c|c|c|c|c|}
\hline PARTICIPANTES & GÉNERO & IDADE & FORMAÇÃO & OCUPAÇÃO \\
\hline$A$ & $M$ & $55-60$ & Medicina & Médico-cirurgião no IPO \\
\hline B & $M$ & $40-45$ & $\begin{array}{l}\text { Doutoramento } \\
\text { Medicina Dentária }\end{array}$ & $\begin{array}{l}\text { Professor na Faculdade de } \\
\text { Medicina Dentária } \\
\text { Desenvolve atividade } \\
\text { clínica }\end{array}$ \\
\hline $\mathrm{C}$ & $M$ & $40-45$ & $\begin{array}{l}\text { Doutoramento } \\
\text { Medicina Dentária }\end{array}$ & $\begin{array}{l}\text { Professor na Escola } \\
\text { Superior de Portalegre } \\
\text { Desenvolve atividade } \\
\text { clínica }\end{array}$ \\
\hline $\mathrm{D}$ & $\mathrm{F}$ & $50-55$ & $\begin{array}{l}\text { Doutoramento } \\
\text { Enfermagem }\end{array}$ & Professora na ESEL \\
\hline$E$ & $\mathrm{~F}$ & $50-55$ & $\begin{array}{l}\text { Doutoranda } \\
\text { Enfermagem }\end{array}$ & Professora na ESEL \\
\hline $\mathrm{F}$ & $\mathrm{F}$ & $35-45$ & $\begin{array}{l}\text { Doutoranda } \\
\text { Enfermagem }\end{array}$ & Professora na ESEL \\
\hline G & $\mathrm{F}$ & $35-45$ & Enfermagem & Enfermeira no HFF \\
\hline $\mathrm{H}$ & $\mathrm{F}$ & $55-60$ & $\begin{array}{l}\text { Enfermagem } \\
\text { Esterilização }\end{array}$ & $\begin{array}{l}\text { Aposentada, formadora } \\
\text { Desenvolve atividade } \\
\text { clínica em voluntariado }\end{array}$ \\
\hline I & $\mathrm{F}$ & $35-45$ & $\begin{array}{ll}\text { Medicina } & \text { Tradicional } \\
\text { Chinesa } & \end{array}$ & $\begin{array}{l}\text { Professora da ESMTC } \\
\text { Desenvolve atividade clínica }\end{array}$ \\
\hline
\end{tabular}

Fonte: Elaboração Própria 
O focus group baseou-se num guião semiestruturado com as seguintes questões: quais as fontes adjuvantes ou oponentes para os profissionais de saúde para o reforço da adesão terapêutica e da relação terapêutica estabelecida; e quais as fontes adjuvantes e oponentes para o paciente para o reforço da sua adesão terapêutica e da relação terapêutica estabelecida. A dinâmica do focus group permitiu uma interação ativa e equilibrada entre os participantes. As respostas a estas questões expressam a perceção destes peritos, isto é, resultaram da avaliação cognitiva destes especialistas sobre as matérias em causa.

\section{Resultados}

Embora se considerar como fonte de informação credível e confiável os profissionais de saúde, os participantes no focus group destacaram amplamente como fonte coadjuvante à relação terapêutica os testemunhos de outras pessoas que têm ou tiveram a mesma doença. Este grupo de pessoas, que experienciaram ou experienciam a doença e partilham de forma significativa as suas "dores" e conquistas, são considerados por estes especialistas como úteis na transmissão de informação e como fontes de informação credível. Estes pares contribuem para uma efetiva educação para a saúde, e há programas que são conduzidos por eles, que têm tido excelentes resultados, referiu a participante $\mathrm{E}$, tendo tido de imediato, a anuência de todo o grupo.

O participante A destacou que existem diferenças entre os pacientes com níveis intermédios de literacia em saúde e os pacientes com níveis baixos de literacia em saúde, sendo que os primeiros são mais seguros porque compreendem e sabem gerir melhor o seu estado de saúde, assim como o contexto onde se encontram. Estes são mais interventivos e assertivos sobre o seu estado de saúde porque têm mais competências para saber gerir a sua saúde.

Já as pessoas com baixa literacia - com baixa capacidade de aceder, usar, compreender e avaliar as informações em saúde (World Health Organization, 1998; Nutbeam, 1998 e 2000; Sorensen, et al., 2012) recorrem ao sistema de saúde (consultas) à procura da autoridade, e muitas vezes nem sequer questionam o profissional, porque têm embaraço em colocar questões, ou por outros motivos, sublinhou o perito $A$, tendo sido confirmado por todo o grupo.

Assinala-se que uma comunicação pobre do paciente e do profissional pode ser uma barreira a adesão ao tratamento e levar a frustração e a comportamentos inadequados (Mathews, Peden, \& Rowles, 2009, p.31). $\mathrm{Na}$ opinião consensual do grupo, as pessoas com alguma literacia em saúde recorrem ao doutor google e apresentam a sua perspetiva, quase de forma imposta aos profissionais de saúde. Esta situação pode gerar, por vezes, um certo conflito entre o profissional de saúde e o paciente. As pessoas que acreditam ou estão convencidas que já têm conhecimento sobre o seu estado de saúde ou doença, e vêm munidas de informação para o profissional de saúde, "muitas vezes perguntam o que não faz sentido nenhum", sublinha o participante $A$.

Na opinião dos participantes, é da competência do profissional saber gerir, controlar e "jogar" com a informação sobre o estado de saúde ou doença, trazida pelos pacientes para o encontro terapêutico. Até porque, "a maioria das centenas de milhões de websites de saúde existentes apresentam informação tendenciosa, pouco clara ou mesmo falsa, levantando questões acerca da utilidade e resultados práticos destas pesquisas" (Carneiro, 2015, p. 5). O grupo concordou que não se pode afastar a informação que 0 
paciente traz para a consulta. Se este pesquisou é porque já teve alguma motivação para o fazer, pelo que a resposta deve permitir a reorientação do paciente.

Por isso, também, o grupo foi unânime em considerar que a internet, apesar das potencialidades positivas, pode ser uma barreira da relação terapêutica entre o profissional de saúde e o paciente.

Existiu também consenso que deve ser o profissional de saúde a verificar se a informação é ou não correta, e contribuir para a educação do paciente esclarecendo-o sobre quais são as fontes fidedignas. Este processo permite que se aumente o nível de literacia e de segurança em saúde. Para que todo este processo de orientação ocorra da melhor forma possível, é fundamental que o profissional tenha um conjunto de competências de comunicação, sublinham os especialistas B e C.

O grupo destacou que a relação terapêutica se baseia num processo dinâmico e que assenta na confiança no outro, numa díade. A relação terapêutica pode aumentar ou diminuir na sua eficácia e conteúdos, dependendo em muitas situações do estado de saúde de quem procura o profissional de saúde. Se uma pessoa estiver numa situação debilitada, vai depositar maior confiança no profissional. Se a pessoa estiver numa situação de controlo, pode não ter tanta confiança no profissional. Depreendemos desta afirmação que a fragilidade física ou emocional do paciente pode aumentar ou diminuir os efeitos /eficácia da relação terapêutica pela maior ou menos confiança por parte do paciente.

O tema da capacitação foi também debatido pelo grupo, e de acordo com a opinião geral do grupo de peritos, esta passa por perceber o que outro quer. 0 elemento de compreensão está patente no processo de capacitação, no âmbito de uma relação terapêutica que se vai construindo.

Quando não existe esclarecimento do paciente durante a relação terapêutica, estes tendem a procurar outras fontes, como os vizinhos, os amigos, a família. O participante $E$, porém, referiu que existe uma clara diferença nas doenças crónicas. Nestas, os pacientes não procuram fontes como os vizinhos ou a internet, salientando que preferem os profissionais de saúde e testemunhos de outros pacientes como fontes mais credíveis. $\mathrm{O}$ participante $\mathrm{E}$ reforçou que os doentes crónicos não procuram os vizinhos nem os amigos para informação sobre a sua doença, e mesmo que saibam utilizar a internet, não recorrem muito à informação contida na internet.

No entanto a opinião foi generalizada para os doentes não crónicos, cujo recurso aos vizinhos e amigos como fontes credíveis de saúde é recorrente, pois apesar de afirmarem em alguns inquéritos realizados, que não recorrem à Internet para pesquisar sobre a sua saúde, "eventualmente por uma questão moral, porque sabem que não o devem fazer" acabam por referir que não recorrem a esta fonte.

Para estes peritos, os media (imprensa, audiovisuais e digitais), são vistos e "sentidos", ao mesmo tempo, como uma balança que oscila como fonte credível e não credível. Todos os participantes consideraram que existem duas facetas, isto é, os media são adjuvantes e ao mesmo tempo oponentes: funcionam para a saúde individual e da população a favor e contra. As fontes que não ajudam os profissionais nem os pacientes são a internet ou os media quando não são bem usados. Têm a mesma opinião sobre a utilização das redes sociais digitais, destacando alguns websites sobre questões de saúde que são falsos, mas em que grande parte da população confia (por exemplo sobre temas como a vacinação das crianças).

Já o participante $\mathrm{H}$ sublinhou e reforçou que em particular a televisão tem ajudado no processo educativo. Considerou que há programas da manhã (especialmente vocacionados para pessoas mais velhas) que fazem educação para a saúde. Os media têm ajudado a educar nas temáticas da saúde da população, por exemplo, abordando temas como o consumo de açúcar. O participante $\mathrm{E}$ acrescentou que tem notado uma maior 
abertura e interesse para as questões de saúde, sobretudo na população mais jovem relacionada com a alimentação saudável. Nas suas palavras, "as pessoas perguntam. Os jovens de 17 anos fazem muitas perguntas sobre os vários assuntos de saúde, o que é um indício positivo".

Para os profissionais de saúde que participaram no focus group, os pares profissionais são fontes coadjuvantes, assim como as boas práticas assentes nos protocolos terapêuticos. Consideram a Direção Geral da Saúde (DGS) como uma boa fonte e coadjuvante, que canaliza as informações das organizações internacionais como o Institute of Medicine.

Entre as fontes adjuvantes, credíveis, o grupo de peritos foi também consensual quando indicou os hospitais de referência, assim como, os resultados da investigação onde se integram as escolas e as universidades. "A boa prática clínica deve ter uma universidade por trás", sublinharam, embora considerassem que toda a investigação tenha de ser temperada pelo olhar crítico do outro, isto é, "são precisas métricas para comparar resultados assim como instrumentos de medida".

A figura 3 resume os resultados obtidos no focus group realizado com a perceção dos especialistas sobre as fontes de informação usadas pelos profissionais de saúde e pelos pacientes e que podem ser adjuvantes e oponentes da relação terapêutica.

Figura 2: Fontes de informação adjuvantes e oponentes da relação terapêutica: a perceção dos profissionais de saúde participantes no focus group

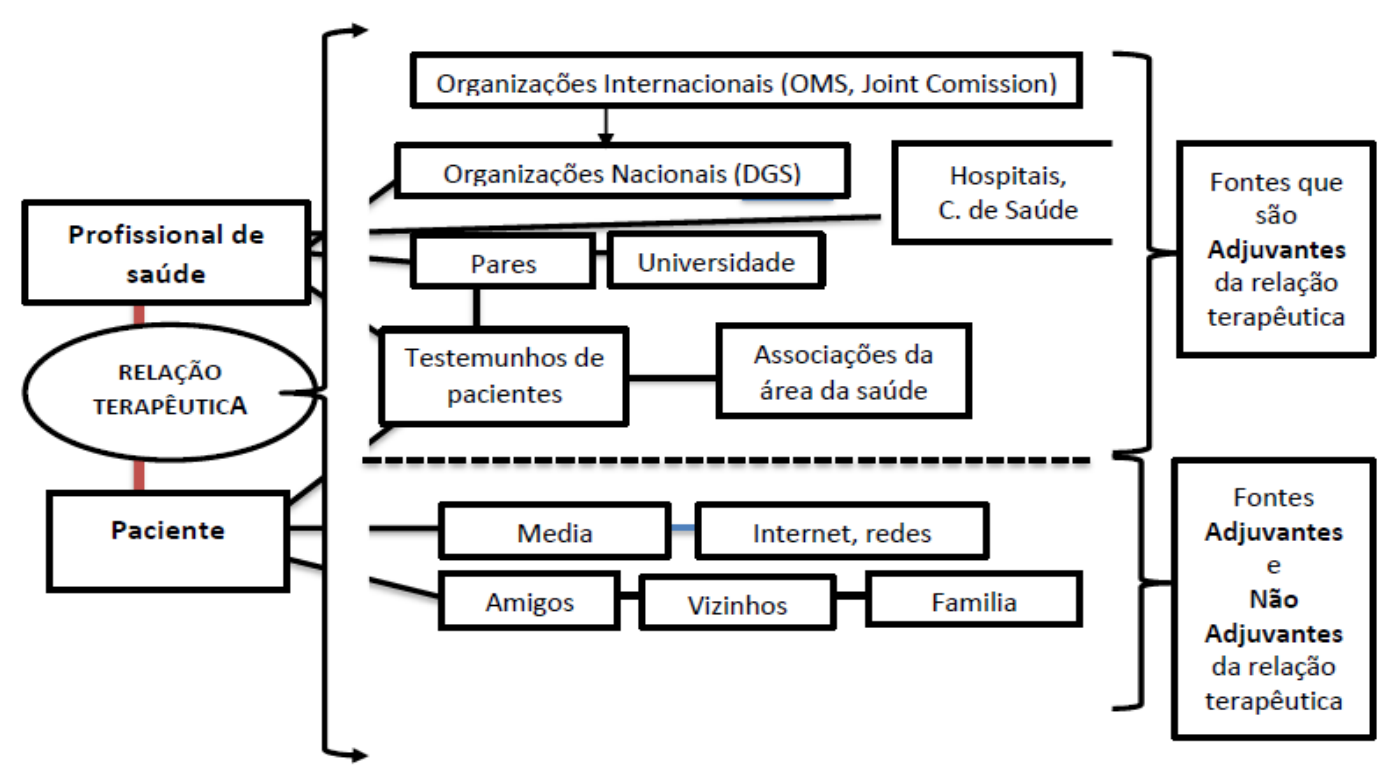

Fonte: Elaboração Própria

\section{Discussão}

Desde o nascimento, as pessoas recebem mensagens de várias fontes, algumas das quais são altamente problemáticas e prejudicam diretamente o comportamento positivo da saúde (Kickbusch, et al., 2013). Para que o recurso a fontes não credíveis não coloque em risco os pacientes, é importante que os profissionais de saúde compreendam o risco de os pacientes não entenderem a informação que lhes é transmitida e que é relevante para a manutenção da saúde (Koh, et al., 2013). Embora com um baixo nível de conhecimento 
e de envolvimento, os pacientes com baixa literacia em saúde e aparentemente "inativos" (Hallahan, 2000), ou latentes (Grunig, 1989; Kim \& Grunig 2011) com estímulos positivos adequados (Kim \& Grunig, 2011), e percebendo de forma consciente a importância da sua ação, melhoram a eficácia na gestão da sua saúde (Bandura, 1986). Neste processo, o aumento das habilidades de comunicação interpessoal dos profissionais de saúde, permite uma dinâmica construtiva da relação terapêutica. Estas competências interrelacionais desenvolvidas através da aprendizagem pelos profissionais de saúde permitem ir ao encontro do grande desafio da obtenção de melhor adesão terapêutica e resultados em saúde. Os pacientes conseguem também baixar o seu nível de constrangimento pela interação que têm com o profissional de saúde através do recurso a fontes credíveis que o podem suportar e apoiar nas suas dúvidas. Os testemunhos dos pares permitem essa credibilização da informação, tanto na ótica dos profissionais como dos pacientes.

Com base na teoria situacional dos públicos (Grunig \& Hunt, 1984) os pacientes têm maior conhecimento do seu processo de doença, por exemplo através dos programas televisivos, que os educam para uma melhor saúde, ou pesquisando na Internet (doutor Google, como referiram os participantes no focus group) sobre as questões relacionadas com a sua saúde ou doença. Estas ações comunicativas promovem a aquisição de informação, recorrendo aos meios (procura e processamento); uma transmissão de informação, especialmente através do reencaminhamento e partilha com os seus pares; e uma seleção de informação (rejeição e aceitação) relativa a dado problema.

$\mathrm{Na}$ opinião dos especialistas do focus group, apesar destes pacientes acederem e recolherem (muitas vezes erradamente) informações sobre saúde e doença na internet, os profissionais de saúde devem incentivar, motivar e também reorientar estas pessoas, num processo de incremento de literacia em saúde, para pesquisas mais fidedignas. Esta ação potencia uma maior eficácia dos resultados da relação terapêutica e consequentemente uma maior adesão do paciente.

O consumo de informação torna-se mais sistemático quando os pacientes vêm que a informação vai ao encontro dos seus problemas (Grunig, 1997), e acabam por investir mais seletivamente os seus recursos cognitivos e comunicativos num problema quando percebem que o seu esforço é necessário e relevante. Os indivíduos tendem assim a ser mais ativos e envolvidos na aquisição, partilha e seleção de informação (Kim \& Grunig, 2011). O reconhecimento do problema (a doença e a pesquisa da mesma através de várias fontes), a diminuição do constrangimento (sobretudo para os pacientes com baixa literacia em saúde, através de uma relação terapêutica baseada na confiança e na autoridade do profissional) e o aumento do envolvimento e da motivação (através da compreensão e reorientação feitas pelos profissionais para as fontes credíveis) permitem este enquadramento nas teorias propostas. Quanto mais ativa se torna a pessoa, mais sistemática, específica e relevante se torna a informação que irá pesquisar e selecionar para a consequente resolução dos problemas (Kim \& Grunig, 2011).

Os profissionais de saúde entrevistados valorizam a pesquisa de informação feita pelos pacientes através dos seus pares referenciadores, isto é, de pessoas que já experienciaram a doença e podem dar um contributo como uma fonte fidedigna. Fazendo um paralelismo com Katz e Lazarsfeld (1955) que realçavam no modelo de comunicação two step flow, a importância das fontes pessoais: os indivíduos, antes de aceitarem como fidedigna uma informação, remetiam-na para os seus pares em quem confiavam e com quem mantinham laços estreitos.

Também os pacientes reforçam a credibilidade da informação (saúde/doença) através da auscultação de testemunhos de outros doentes, que servem de referência credível. A credibilidade destes testemunhos é 
sentida tanto pelos pacientes como pelos próprios profissionais de saúde, de acordo com a reflexão do focus group. O próprio profissional de saúde é visto como uma "autoridade" nesta relação interpessoal comunicacional e colaborativa, e gere, de forma predominante, a relação terapêutica e a informação, em especial com pacientes que possuem baixa literacia em saúde.

\section{Conclusões}

Neste estudo evidencia-se a importância da aplicação da teoria da resolução dos problemas (Kim \& Grunig, 2011) na avaliação das dinâmicas e dos contributos comunicacionais para a relação terapêutica, especialmente com pacientes com baixa literacia em saúde e a abordagem da capacitação dos profissionais de saúde no processo de comunicação ao paciente. De acordo com o objetivo de aferição da perceção dos profissionais de saúde sobre a importância das fontes de informação para a relação terapêutica, o estudo confirmou-se que existem fontes diferenciadas credíveis e não credíveis para ambos os públicos analisados: profissionais de saúde e pacientes. Tanto o profissional de saúde como o paciente devem recorrer a fontes de informação de confiança, credíveis e competentes. No entanto, sublinha-se que os profissionais de saúde têm uma relevância e responsabilidade acrescida na relação terapêutica, particularmente na gestão dos aspetos comunicacionais, sendo esta competência crucial para a orientação e motivação dos pacientes.

É também relevante que o acesso à informação sobre saúde na internet acarreta vários riscos, em particular os pacientes com baixa literacia em saúde, necessitando por isso de ser vista como uma balança informativa com vários pesos, onde o profissional tem um papel crítico na orientação acertada para o objetivo de resolução dos problemas. Por conseguinte, recomenda-se que se exerça uma auditoria mais eficaz relativamente aos conteúdos em saúde para garantir a disponibilização online de informações de saúde de alta qualidade, permitindo aos profissionais de saúde direcionar os pacientes para essas fontes.

Este processo implica também a formação dos profissionais de saúde em comunicação e aconselhamento dos pacientes sobre o uso de fontes credíveis e não credíveis, para aceder a informações sobre fatores de risco, prevenção, promoção da saúde, diagnóstico e tratamento, como forma de se criar um melhor conhecimento e envolvimento dos pacientes que promovam a melhor resolução de problemas (ex. adesão terapêutica) e por isso uma melhor literacia em saúde do cidadão.

O reforço do comportamento comunicacional dos interlocutores de saúde, tornando-os mais ativos na aquisição, seleção e transmissão da informação aumenta o compromisso na relação terapêutica e o seu objetivo maior de resolver "problemas", tanto do paciente que está numa situação de fragilidade, como do profissional que pretende resultados em saúde.

Apesar dos aspetos subjetivos e emocionais que rodeiam o testemunho de pares (pacientes que sofrem ou sofreram da mesma doença ou estado de saúde) foi considerada uma fonte muito credível, tanto pelo grupo de especialistas como pela literatura. Estes grupos de autoajuda (com testemunhos de doentes ou de pessoas que passaram pelo mesmo processo de fragilidade) estão cada vez mais a ser reconhecidos como métodos viáveis e eficientes de complementar e ampliar o atual sistema de saúde e cuidados de saúde mental, a ponto de serem caracterizados como um movimento social emergente (Heisler, 2006; Katz, 1981). Considera-se que a matéria relativa as fontes de informação credíveis e não credíveis é por si só ampla e quase inesgotável, havendo por isso margem para se prosseguir naquilo que foi uma abordagem controlada 
e limitada a um conjunto de questões muito direcionadas para a reflexão de fontes credíveis e não credíveis para estes elementos do processo da relação terapêutica, profissional e paciente. Estudos futuros poderão, por conseguinte, alargar a participação a profissionais de saúde menos sensibilizados para a questão da literacia em saúde e das competências comunicacionais, assim como, à recolha de opiniões junto dos pacientes.

\section{Referências Bibliográficas}

Aldoory, L. \& Sha, B. (2007). The situation theory of publics: practical applications, methodological challenges and theoretical horizons. In J. E. Grunig, L. A. Grunig, \& E. L. Toth, The future of excellence in public relations and communication management: challenges for the next generation (pp. 339-355). Mahwah: Lawrence Erlbaum.

Anderson, R. B., \& McMillion, P. Y. (1995). Effects of similar and diversified modeling on African American women's efficacy expectations and intentions to perform breast self-examination. Health Communication, 7(4), 327-343. doi: 10.1207/s15327027hc0704_3

Andreassen, H. K., Bujnowska-Fedak, M. M., Chronaki, C., Dumitru, R. C., Pudule, I., Santana, S. ... \& Wynn, R. (2007). European citizens' use of e-health services: A study of seven countries, BMC Public Health, 7(1), 53-59. doi: 10.1186/1471-2458-7-53

Bandura, A. (1986). Social foundations of thought and action: A social cognitive theory. Englewood Cliffs: Prentice-Hall.

Berlo, D. K. (1985). O processo de comunicação: Introdução à teoria e à prática (5a edição). São Paulo: Martins Fontes.

Blumer, H. (1966). The mass, the public, and public opinion. In B. Berelson \& M. Janowitz (Eds.), Reader in public opinion and communication (pp. 43-50). New York: Free Press.

Carneiro, A.,V., (2015) Literacia em saúde: desenvolvimento de um sistema de informação em saúde para pacientes: Relatório de projecto. Disponível via Centro de Estudos de Medicina Baseada na Evidência da Faculdade de Medicina da Universidade de Lisboa em: http://cembe.org/avc/docs/Projecto\%20de\%20Literacia\%20em\%20Sa\%C3\%BAde\%202015.pdf

Cassell, E.J. (1985). Talking with patients. (Vol. 2). Cambridge: MIT Press.

Chaiken, S. (1980). Heuristic versus systematic information processing and the use of source versus message cues in persuasion. Journal of Personality and Social Psychology. 39, 752-766.

Cegala, D. J., McNeilis, K. S., \& McGee, D. S. (1995). A study of doctors and patients perceptions of information processing and communication competence during the medical interview. Health Communication, 7(3), 179-203.

Chew, M., S-Y. (2001). Rational Ritual: Culture coordination and common knowledge. Princeton: Princeton University Press.

Chinn, D. A., \& McCarthy, C. (2012). All aspects of health literacy scale (AAHLS): Developing a tool to measure functional, communicative and critical health literacy in primary healthcare settings. Patient Education Counseling, 90(2), 247-253. doi: 10.1016/j.pec.2012.10.019 
Cutilli, C. C. (2010). Seeking health information: what sources do your patients use? Orthopaedic Nursing, 29(3), 214-219. doi: 10.1097/NOR.0b013e3181db5471

Dewey, J. (1927). The public and its problems. Chicago: Swallow Press.

Espanha, R., Ávila, P. \& Mendes, R. V. (2016). Relatório Síntese - Literacia em Saúde em Portugal. Lisboa: Fundação Calouste Gulbenkian. Disponível em: https://gulbenkian.pt/publication/literacia-emsaude-em-portugal/

Ghaddar, S. F., Valerio, M. A., Garcia, C. M., \& Hansen, L. (2012). Adolescent health literacy: The importance of credible sources for online health information. Journal of School Health, 82 (1), 28-36.

Gray, N. J, Klein, J. D., Noyce, P. R., Sesselberg, T. S., \& Cantrill, J. A. (2005). Health information-seeking behavior in adolescence: the place of the Internet. Social Science \& Medicine, 60, 1467-1478.

Grunig, J. E. (2006). Furnishing the edifice: Ongoing research on public relations as a strategic management function. Journal of Public Relations Research, 18, 151-176.

Grunig, J. E. (1997). A situational theory of publics: Conceptual history, recent challenges and new research. In D. Moss, T. MacManus, \& D. Vercic (Eds.), Public relations research: An international perspective (pp. 3-48). London: International Thomson Business Press.

Grunig, J. E., \& Hunt, T. (1984). Managing public relations. New York: Holt, Rinehart \& Winston.

Grunig, J. E., \& Repper, F. C. (1992). Strategic management, publics and issues. In J. E. Grunig (Ed.), Excellence in public relations and communication management (pp. 117-157). New Jersey: Lawrence Erlbaum.

Hallahan, K. (2000). Inactive Publics: The forgotten publics in public relations. Public Relations Review, 26 (4), 499-515.

Heisler, M. (2006). Building peer support programs to manage chronic disease: Seven models for success. Oakland \& Sacramento: California Health Care Foundation.

Isaacs, S.L. (1996). Consumer's information needs: results of a national survey. Health Affairs, 15(4), 3141. doi: $10.1377 /$ hlthaff.15.4.31

Hunt, T., \& Grunig, J. E. (1994). Public relations techniques. Fort Worth: Harcourt Brace College Publishers. Jackson, L. D. (1992). Information complexity and medical communication: The effects of technical language and amount of information in a medical message. Health Communication, 4(3), 197-210. doi: 10.1207/s15327027hc0403_3

Kickbusch, I., Pelikan, J., M., Apfel, F., \& Tsouros, A. D. (Ed.) (2013). Health literacy: the solid facts. World Health Organization. Disponível em: http://www.euro.who.int/_data/assets/pdf_file/0008/190655/e96854.pdf

Katz, E., \& Lazarsfeld, P. (1955). Personal influence: the part played by people in the flow of mass communication. Glencoe: Free Press.

Katz, A. (1981). Self-help and mutual aid: An emerging social movement? Annual Review of Sociology, 7, 129-155.

Keltner D., Oatley K., \& Jenkins, J.M. (2014). Understanding emotions. Hoboken: Wiley.

Kim, J-N., Ni, L., Kim, S-H., \& Kim, J. R. (2012). What makes people hot? Applying the situational theory of problem solving to hot-issue publics. Journal of Public Relations Research, 24(2), 144-164.

Kim, J.-N., Ni, L., \& Sha, B.-L. (2008). Breaking down the stakeholder environment: A review of approaches to the segmentation of publics. Journalism \& Mass Communication Quarterly, 85(4), 751-768. 
Kim, J-N., \& Grunig, J. E. (2011). Problem solving and communicative action: A situational theory of problem solving. Journal of Communication, 61, 120-149.

Koh, H. K., Brach, C., Harris, L. M., \& Parchman, M. L. (2013). A proposed 'health literate care model' would constitute a systems approach to improving patients' engagement in care. Health Affairs Journal, 32(2), 357-367. doi: 10.1377/hlthaff.2012.1205

Kripalani, S., Bentgtzen, R., Henderson, L. E., \& Jacobson, T. A. (2008). Clinical research in low literacy population: using teach back to assess comprehension on informed consent and privacy information. Ethics and Human Research, 30(2), 13-19.

Mathews, S. M., Peden, A. R., \& Rowles, G. D. (2009). Patient-provider communication: Understanding diabetes management among adult females. Patient Education and Counseling, 76, 31-37.

Moon, B. B., Rhee, Y., \& Yang, S-U. (2016). Developing public's information transmitting behavior (ITB) model in public relations: a cross national study. Journal of Public Relations Research, 28(1), 4-18.

Mukherjee, A., \& Bawden, D. (2012). Health information seeking in the information society. Health Information and Libraries Journal, 29(3), 242-246. doi: 10.1111/j.1471-1842.2012.00997

Mutz, D. (1998). Impersonal influence: how perceptions of mass collectives affect political attitudes. Cambridge: Cambridge University Press.

Norman, C. D., \& Skinner, H., A., (2006). Ehealth: The ehealth literacy scale. Journal of Medical Internet Research, 8(4), 1-7. doi: 10.2196/jmir.8.4.e27

Nutbeam, D. (1998). Health promotion Glossary. Health Promotion International, 13(4), 349-364.

Nutbeam, D. (2000). Health literacy as public goal: a challenge for contemporary health education and communication strategies into de $21^{\text {st }}$ century. Health Promotion International, 15(3), 259-267. doi:10.1093/heapro/15.3.259

Organização Mundial da Saúde (1986). Carta de Ottawa para a promoção da saúde. In I conferência internacional sobre promoção da saúde, Ottawa, 17-21 novembro de 1986. Disponível em: https://www.dgs.pt/paginas-de-sistema/saude-de-a-a-z/carta-de-otawa-1986.aspx

Pinto, M. (2000). Fontes jornalísticas: contributos para o mapeamento do campo. Comunicação e Sociedade 2, Cadernos do Noroeste, Série Comunicação, 14(1-2), 277-294.

Ritchie, J. \& Lewis, J. (Eds).(2003). Qualitative research practice a guide for social science students and researchers. London: Sage Publications

Rouner, D. (2008). Credibility of content. In W. Donsbach, The international encyclopedia of communication (pp. 1039-1044). Malden: Blackwell Publishing.

Saboga-Nunes, L. (2014). Literacia para a saúde e a conscientização da cidadania positiva. Revista Referencia, III Serie (Supl.), 94-99.

Simou, E. (2016). Health information sources: trust and satisfaction. International Journal of Healthcare, 2(1), 38-43. doi: 10.5430/ijh.v2n1p38

Skinner, H., Biscope, S., Poland, B., \& Goldberg, E. (2004). How adolescents use technology for health information: implications for health professionals from focus group studies. Journal of Medical Internet Research, 18(5), 4-32. doi: 10.2196/jmir.5.4.e32

Sørensen, K., Broucke, V., Fullam J., Doyle, G., Pelikan, J. Slonska, Z., \& Brand, H. Consortium Health Literacy Project European (2012). Health literacy and public health: A systematic review and integration of definitions and models. BMC Public Health, 12, 80. doi:10.1186/1471-2458-12-80 
Stellefson, M., Hanik, B., Chaney, B., Chaney, D., Tennant, B., \& Chavarria, E. A. (2011). Ehealth literacy among college students: a systematic review with implications for ehealth education. Journal of Medical Internet Research, 13(4), e102.

Vaz de Almeida, C. (2016). Literacia em Saúde: Os caminhos para uma maior capacitação dos profissionais de saúde. ACE Associação Científica dos Enfermeiros. Suplemento Enformação: Congresso Acolher, Capacitar, Encaminhar (Auditório do Alto dos Moinhos, Lisboa).

Viswanath, K. (2008). Health communication. In W. Donsbach, The international encyclopedia of communication (pp. 2073-2087). Malden: Blackwell Publishing.

Williams, M. V., Davis, T., Parker, R. M., \& Weiss, B. D. (2002). The role of health literacy in patient-physician communication. Family Medicine, 34(5), 383-389. Disponível em: http://www.stfm.org/Portals/49/Documents/FMPDF/FamilyMedicineVol34Issue5Williams383.pdf

Wilkinson, R., \& Marmot, M. (Ed.) (2003). Social determinants of health: the solid facts (2 ${ }^{\text {nd }}$ ed). Copenhagen: Who.

World Health Organization (1998). Health promotion glossary. Disponível em: http://www.who.int/healthpromotion/about/HPR\%20Glossary\%201998.pdf 